\title{
Reply - Aortic Reservoir Pressure - not overstretching but testing
}

\author{
Cameron, James, D. ${ }^{\text {a; }}$ Parker, Kim ${ }^{\text {; }}$ Narayan, Omc; Davies, Justin ${ }^{\text {d }}$; Hughes, Alun ${ }^{\mathrm{e}}$
}

We thank Drs Westerhof, Westerhof and Segers for their interest ${ }^{[1]}$ in our recent report ${ }^{[2]}$. We welcome their comment that '...there is not the slightest doubt that reservoir pressure waveforms are similar ... .' This is the main point of our article, and in the accompanying editorial this was considered to confirm one of the cornerstones of the reservoir pressure concept ${ }^{[3]}$.

The correspondents are incorrect in saying that no statistical tests are reported - the results of linear mixed model analyses are given in Tables 3-5 and shown in Fig. 3. We have, however, in accordance with recent statistical guidelines, not presented $P$ values ${ }^{[4]}$. On the basis of these data, there is no convincing evidence that the within-individual variability of reservoir pressure and measured pressure differ. For example, the intraclass correlation coefficient (ICC, a measure of within-individual similarity between sites) for maximum $P_{\mathrm{r}}$ - diastolic pressure (Table 5) is 0.98 [95\% confidence interval (CI) 0.96-0.99], whereas for SBP (Table 3 ), it is 0.96 (95\% CI $0.94-0.98 ; 95 \%$ CIs of the ICCs were omitted from the original tables because of space limitations).

With regard to their second point regarding the assumption that $P x(x, t)=\xi Q$ in $(t)$. This has

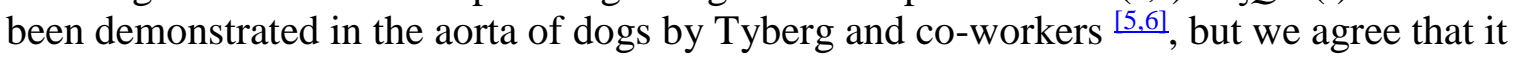
may not apply in all locations. The data that they show are consistent with this expectation, and our recent data ${ }^{[7]}$. We believe the correspondents' suggestion regarding the goodness of fit of the reservoir model and the 'Windkesselness' of the system studied is credible, but should be tested further experimentally. It is also worth emphasizing that, although cursory consideration may lead to the conclusion that variation along the aorta in the parameters used to calculate $P_{\mathrm{r}}\left(k_{\mathrm{d}}, k_{\mathrm{s}}\right.$, and $\left.P_{\mathrm{inf}}\right)$ is evidence against the assumption that $P_{\mathrm{r}}$ is uniform along the aorta, given the obvious variation in $P$ meas at the different aortic sites, it is in fact necessary that these parameters are different if $P_{\mathrm{r}}$ is to be constant.

Importantly we do not, and have not, claimed that $\xi$ is a characteristic impedance (although as discussed in our article ${ }^{[2]}$, pages 3 and 4 of Supplementary File 2, it has the dimensions of impedance). In our view, $\xi$ is best considered as an heuristic parameter determined through fitting ( $k_{\mathrm{s}}=1 / \xi C$, where $C$ is the compliance). With regard to the correspondents' point 3 , we regret if the use of data shown in Fig. 1 muddied the waters. Figure 1 was only used as a schematic illustration to define the various parameters that we calculated in our study and is not from the data set analysed.

We note Drs Westerhof, Westerhof and Segers' point 4 concerning the relationship between $P_{\mathrm{r}}$ and a backward pressure wave $\left(P_{\mathrm{b}}\right)$ but feel we have adequately presented this and discussed it at length in the original Supplementary File 2. There we pointed out some problems with the generalization of this concept but did acknowledge that $P_{\mathrm{r}}=2 P_{\mathrm{b}}$ obtains in diastole in the aorta. Other points raised seem to relate to the previously mentioned issues around the interpretation of $\xi$, and its relation to impedance - characteristic or otherwise. Clearly aortic root input impedance is not the same as the local characteristic impedance and characteristic impedance is not space-invariant in the circulation. Additionally, a frequency domain derivation of impedance is not necessarily identical to a local time-domain estimate. 
We consider the characteristic impedance to be represented as $z=\mathrm{d} P(t) / \mathrm{d} Q(t)$ for a single wave front (which should be carefully distinguished from the estimate of characteristic impedance, $Z_{0}(\omega)$, obtained by Fourier analysis and calculated from $P(\omega)$ and $Q(\omega)$ ). In this definition $z$ is a real local parameter (that may be a function of time) and depends on the density of blood and the area and distensibility of the local artery; $z=Z_{0}$ whenever there are no reflections in the arterial system.

Finally, the correspondents' comment '... magnitude and shape of Pr are still assumed similar at all locations (their italics). Narayan et al. now attempt to underpin this assumption' leads us to re-emphasize that the purpose of the study was to test the hypothesis that $P_{\mathrm{r}}$ is similar at all locations. Clearly we did not attempt to 'underpin' an assumption but to 'test' it.

We trust that these comments will help in providing further background and an expanded context in which our results can be interpreted. 